Ferhan M. Atıcı • Hatice Yaldız

\title{
Refinements on the discrete Hermite-Hadamard inequality
}

Received: 7 September 2017 / Accepted: 3 December 2017 / Published online: 12 December 2017

(C) The Author(s) 2017. This article is an open access publication

Abstract In this paper, we use techniques and tools from time scale calculus to state and prove many refinements on the discrete Hermite-Hadamard inequality.

Mathematics Subject Classification $\quad 26 \mathrm{D} 10 \cdot 26 \mathrm{D} 15 \cdot 26 \mathrm{~A} 33 \cdot 39 \mathrm{~A} 12 \cdot 39 \mathrm{~A} 70$

في هذه المقالة نستخدم تقنيات وأدوات المقياس الزمني لنصوغ ونبرهن عدة تحسينات لمتراجحة هيرميت-هدمارد

\section{Introduction}

The Hermite-Hadamard inequality $[9,10]$ states that if $f: I \rightarrow \mathbb{R}$ is a convex function, then the following inequality is satisfied:

$$
f\left(\frac{a+b}{2}\right) \leq \frac{1}{b-a}\left(\int_{a}^{b} f(t) \mathrm{d} t\right) \leq \frac{f(a)+f(b)}{2},
$$

where $a, b \in I$ and $I$ is an interval in $\mathbb{R}$. Hermite-Hadamard's inequality for convex functions has received renewed attention in recent years and a remarkable variety of refinements and generalizations have been found (see, for example, [3-6,12,13]).

Regarding the definition of a convex function on a time scale, a pioneering work has been done by Mozyrska and Torres [11]. They introduced the convexity of a function defined on a time scale (a nonempty closed subset of $\mathbb{R}$ ) and proved a theorem which characterizes the convex function in terms of its second derivative.

Since the midpoint condition plays an important role in the proofs related to the Hermite-Hadamard inequality, the authors first defined the midpoint condition for the functions on the set of integers, $\mathbb{Z}$, in [1]. Then, some equivalent conditions for convexity have been given, and the discrete Hermite-Hadamard inequality has been proven.

F. M. At1c1

Department of Mathematics, Western Kentucky University, Bowling Green, KY 42101-3576, USA

E-mail: ferhan.atici@wku.edu

H. Yaldız ( $\varangle)$

Department of Mathematics, Kamil Özdağ Science Faculty, Karamanoğlu Mehmetbey University, Yunus Emre Campus,

70100 Karaman, Turkey

E-mail: yaldizhatice@gmail.com 
Let $a, b \in \mathbb{Z}$ with $a<b .[a, b]_{\mathbb{Z}}$ means $[a, b] \cap \mathbb{Z}$. We define

$$
\mathbb{T}_{[a, b]}=\left\{u \mid u=\frac{t-b}{a-b} \quad \text { for } t \in[a, b]_{\mathbb{Z}}\right\} .
$$

We note that $\mathbb{T}_{[a, b]}$ is a subset of the real interval $[0,1]$. Since the set $\mathbb{T}_{[a, b]}$ is an isolated time scale, from now on we use the notations of the time scale calculus.

Definition 1.1 [1] $f: \mathbb{Z} \rightarrow \mathbb{R}$ is called convex on $\mathbb{Z}$ if for every $x, y \in \mathbb{Z}$ with $x<y$ the following inequality

$$
f(\lambda x+(1-\lambda) y) \leq \lambda f(x)+(1-\lambda) f(y)
$$

is satisfied for all $\lambda \in \mathbb{T}_{[x, y]}$.

Theorem $1.2[1]$ Suppose $f: \mathbb{Z} \rightarrow \mathbb{R}$ is a convex function on $[a, b]_{\mathbb{Z}}$ with $a, b \in \mathbb{Z}$ and $a<b$, and $a+b$ an even number. Then

$$
f\left(\frac{a+b}{2}\right) \leq \frac{1}{2(b-a)}\left[\int_{a}^{b} f(t) \Delta t+\int_{a}^{b} f(t) \nabla t\right] \leq \frac{f(a)+f(b)}{2} .
$$

Our aim in this paper is to continue our work on the discrete Hermite-Hadamard inequality. We state and prove some refinements on both sides of the inequality (4).

The plan of the paper is as follows: In Sect. 2, we list the integration by parts formulas and the substitution rules for integrals on time scales. For the purpose of comparison, the continuous case and the discrete case, we list the refinements obtained for the continuous Hermite-Hadamard inequality. In Sect. 3, we state and prove some refinements related to both sides of the discrete Hermite-Hadamard inequality.

\section{Preliminaries}

The following two theorems will play important roles in the proof of our main results.

Theorem 2.1 (Integration by parts) [2] If $a, b, c \in \mathbb{T}$ and $f, g \in \mathbb{C}_{r d}$, then

$$
\begin{aligned}
& \int_{a}^{b} f(\sigma(t)) g^{\Delta}(t) \Delta t=(f g)(b)-(f g)(a)-\int_{a}^{b} f^{\Delta}(t) g(t) \Delta t \\
& \int_{a}^{b} f(\rho(t)) g^{\nabla}(t) \nabla t=(f g)(b)-(f g)(a)-\int_{a}^{b} f^{\nabla}(t) g(t) \nabla t .
\end{aligned}
$$

Theorem 2.2 (Substitution rule) $[2,8]$ Let $v: \mathbb{T} \rightarrow \mathbb{R}$ be monotone.

(i) Assume $v$ is strictly increasing and $\widetilde{\mathbb{T}}: v(\mathbb{T})$ is a time scale. If $f: \mathbb{T} \rightarrow \mathbb{R}$ is a an rd-continuous function and $v$ is differentiable with rd-continuous derivative, then if $a, b \in \mathbb{T}$,

$$
\int_{a}^{b} f(t) v^{\Delta}(t) \Delta t=\int_{\nu(a)}^{v(b)}\left(f \circ v^{-1}\right)(s) \widetilde{\Delta} s
$$

or

$$
\int_{a}^{b} f(t) v^{\nabla}(t) \nabla t=\int_{\nu(a)}^{\nu(b)}\left(f \circ v^{-1}\right)(s) \widetilde{\nabla} s
$$


(ii) Assume $v$ is strictly decreasing and $\widetilde{\mathbb{T}}:-v(\mathbb{T})$ is a time scale. If $f: \mathbb{T} \rightarrow \mathbb{R}$ is an rd-continuous function and $v$ is differentiable with rd-continuous derivative, then if $a, b \in \mathbb{T}$,

$$
\int_{a}^{b} f(t) v^{\Delta}(t) \Delta t=\int_{\nu(a)}^{\nu(b)}\left(f \circ v^{-1}\right)(s) \widetilde{\nabla} s
$$

or

$$
\int_{a}^{b} f(t) v^{\nabla}(t) \nabla t=\int_{v(a)}^{v(b)}\left(f \circ v^{-1}\right)(s) \widetilde{\Delta} s
$$

In [7], Dragomir and Agarwal proved the following results connected with the right part of (1).

Lemma 2.3 Let $f: I^{\circ} \subseteq \mathbb{R} \rightarrow \mathbb{R}$ be a differentiable mapping on $I^{\circ}, a, b \in I^{\circ}$ with $a<b$. If $f^{\prime} \in L[a, b]$, then the following equality holds:

$$
\frac{f(a)+f(b)}{2}-\frac{1}{b-a} \int_{a}^{b} f(x) \mathrm{d} x=\frac{b-a}{2} \int_{0}^{1}(1-2 t) f^{\prime}(t a+(1-t) b) \mathrm{d} t .
$$

Theorem 2.4 Let $f: I^{\circ} \subseteq \mathbb{R} \rightarrow \mathbb{R}$ be a differentiable mapping on $I^{\circ}, a, b \in I^{\circ}$ with $a<b$. If $\left|f^{\prime}\right|$ is convex on $[a, b]$, then the following equality holds:

$$
\left|\frac{f(a)+f(b)}{2}-\frac{1}{b-a} \int_{a}^{b} f(x) \mathrm{d} x\right| \leq \frac{b-a}{8}\left(\left|f^{\prime}(a)\right|+\left|f^{\prime}(b)\right|\right) .
$$

\section{Main results}

In this section, we start with stating and proving the discrete counterparts of Lemma 2.3 and Theorem 2.1. Then, some other refinements will follow.

Lemma 3.1 Suppose $f: \mathbb{Z} \rightarrow \mathbb{R}$ is a function. Let $a, b \in \mathbb{Z}$ with $a<b$ and $a+b$ be an even number. Then

$$
\begin{aligned}
& {\left[\frac{f(a)+f(b)}{2}-\frac{1}{2(b-a)}\left(\int_{a}^{b} f(t) \Delta t+\int_{a}^{b} f(t) \nabla t\right)\right]} \\
& \left.=\frac{b-a}{4}\left[\int_{0}^{1}(1-2 \widetilde{\rho}(t)) f^{\Delta}(t a+(1-t) b) \widetilde{\nabla} t-\int_{0}^{1}(1-2 \widetilde{\rho}(t)) f^{\nabla}((1-t) a+t b)\right) \widetilde{\nabla} t\right] .
\end{aligned}
$$

Proof First, we claim that

$$
\frac{b-a}{4} \int_{0}^{1}(1-2 \widetilde{\rho}(t)) f^{\Delta}(t a+(1-t) b) \widetilde{\nabla} t=\frac{f(a)+f(b)}{4}-\frac{1}{2(b-a)} \int_{a}^{b} f(u) \Delta u .
$$

To prove our first claim, we first use substitution method given in (Theorem 2.2) and then use integration by parts formula (Theorem 2.1 ). Here, we have $v(t)=\frac{b-t}{b-a}$.

We apply the substitution method (Theorem 2.2) for the integral

$$
\int_{0}^{1}(1-2 \widetilde{\rho}(t)) f^{\Delta}(t a+(1-t) b) \widetilde{\nabla} t
$$


to get that

$$
\begin{aligned}
& \int_{0}^{1}(1-2 \widetilde{\rho}(t)) f^{\Delta}(t a+(1-t) b) \widetilde{\nabla} t \\
& =\frac{1}{b-a} \int_{a}^{b}\left(1-2\left(\frac{b-\sigma(u)}{b-a}\right)\right) f^{\Delta}(u) \Delta u,
\end{aligned}
$$

where $g(t)=\left(1-2\left(\frac{b-\sigma(t)}{b-a}\right)\right),\left(g \cdot f^{\Delta}\right)\left(v^{-1}(t)\right)=\left(1-2\left(\frac{b-\sigma\left(v^{-1}(t)\right)}{b-a}\right)\right) f^{\Delta}\left(v^{-1}(t)\right)$. Now, we apply integration by parts to the last integral, we have

$$
\begin{aligned}
& =\frac{1}{b-a} \int_{a}^{b}\left(1-2\left(\frac{b-\sigma(u)}{b-a}\right)\right) f^{\Delta}(u) \Delta u \\
& =\frac{1}{b-a}\left\{\left.\left(1-2\left(\frac{b-u}{b-a}\right)\right) f(u)\right|_{a} ^{b}-\int_{a}^{b} \frac{2}{b-a} f(u) \Delta u\right\} \\
& =\frac{1}{b-a}\left\{f(a)+f(b)-\frac{2}{b-a} \int_{a}^{b} f(u) \Delta u\right\} .
\end{aligned}
$$

If we multiply the last quantity by $\frac{b-a}{4}$, we have the desired result.

Next, we claim that

$$
-\frac{b-a}{4} \int_{0}^{1}(1-2 \widetilde{\rho}(t)) f^{\nabla}((1-t) a+t b) \widetilde{\nabla} t=\frac{f(a)+f(b)}{4}-\frac{1}{2(b-a)} \int_{a}^{b} f(t) \nabla t .
$$

To prove this claim, we first use the substitution rule (Theorem 2.2) and then use integration by parts formula (Theorem 2.1). Here, we have

$$
\begin{aligned}
& -\int_{0=v(a)}^{1=v(b)}(1-2 \widetilde{\rho}(t)) f^{\nabla}((1-t) a+t b) \widetilde{\nabla} t \\
& =-\frac{1}{b-a} \int_{a}^{b}\left(1-2\left(\frac{\rho(u)-a}{b-a}\right)\right) f^{\nabla}(u) \nabla u,
\end{aligned}
$$

where $v(t)=\frac{t-a}{b-a}$.

Next, we use the integration by parts formula to the last integral above. We have

$$
\begin{aligned}
& -\frac{1}{b-a} \int_{a}^{b}\left(1-2\left(\frac{\rho(u)-a}{b-a}\right)\right) f^{\nabla}(u) \nabla u \\
& =-\frac{1}{b-a}\left\{\left.\left(1-2\left(\frac{u-a}{b-a}\right)\right) f(u)\right|_{a} ^{b}+\int_{a}^{b} \frac{2}{b-a} f(u) \nabla u\right\} \\
& =\frac{1}{b-a}\left\{f(a)+f(b)-\frac{2}{b-a} \int_{a}^{b} f(u) \nabla u\right\} .
\end{aligned}
$$

Adding (7) and (8) side by side, we have the desired result. 
Theorem 3.2 Suppose $f: \mathbb{Z} \rightarrow \mathbb{R}$ is a function. Let $a, b \in \mathbb{Z}$ with $a<b$, and $a+b$ be an even number. If $\left|f^{\Delta}\right|$ and $\left|f^{\nabla}\right|$ are convex on $[a, b]_{\mathbb{Z}}$, then the following inequality holds:

$$
\begin{aligned}
& \left|\frac{f(a)+f(b)}{2}-\frac{1}{2(b-a)}\left(\int_{a}^{b} f(t) \Delta t+\int_{a}^{b} f(t) \nabla t\right)\right| \\
& \leq \frac{b-a}{4}\left[\left|f^{\Delta}(a)\right| \frac{1}{4}-\left|f^{\Delta}(b)\right| \frac{1}{4}-\left|f^{\nabla}(a)\right| \frac{1}{4}+\left|f^{\nabla}(b)\right| \frac{1}{4}\right] .
\end{aligned}
$$

Proof Using Lemma (3.1) and the convexity on $[a, b]_{\mathbb{Z}}$ of $\left|f^{\Delta}\right|$ and $\left|f^{\nabla}\right|$, we obtain

$$
\begin{aligned}
\mid & \frac{f(a)+f(b)}{2}-\frac{1}{2(b-a)}\left(\int_{a}^{b} f(t) \Delta t+\int_{a}^{b} f(t) \nabla t\right) \mid \\
= & \frac{b-a}{4}\left|\int_{0}^{1}(1-2 \widetilde{\rho}(t)) f^{\Delta}(t a+(1-t) b) \widetilde{\nabla} t+\int_{0}^{1}(2 \widetilde{\rho}(t)-1) f^{\nabla}((1-t) a+t b) \widetilde{\nabla} t\right| \\
\leq & \frac{b-a}{4}\left|\int_{0}^{1}\right| 1-2 \widetilde{\rho}(t)|| f^{\Delta}(t a+(1-t) b)\left|\widetilde{\nabla} t+\int_{0}^{1}\right| 2 \widetilde{\rho}(t)-1|| f^{\nabla}((1-t) a+t b)|\widetilde{\nabla} t| \\
\leq & \frac{b-a}{4}\left[\int_{0}^{1}|1-2 \widetilde{\rho}(t)|\left[t\left|f^{\Delta}(a)\right|+(1-t)\left|f^{\Delta}(b)\right|\right] \widetilde{\nabla} t\right. \\
& \left.+\int_{0}^{1}|1-2 \widetilde{\rho}(t)|\left[(1-t)\left|f^{\nabla}(a)\right|+t\left|f^{\nabla}(b)\right|\right] \widetilde{\nabla} t\right] \\
= & \frac{b-a}{4}\left[\left|f^{\Delta}(a)\right| \int_{0}^{1}|1-2 \widetilde{\rho}(t)| t \widetilde{\nabla} t+\left|f^{\Delta}(b)\right| \int_{0}^{1}|1-2 \widetilde{\rho}(t)|(1-t) \widetilde{\nabla} t\right. \\
& \left.+\left|f^{\nabla}(a)\right| \int_{0}^{1}|1-2 \widetilde{\rho}(t)|(1-t) \widetilde{\nabla} t+\left|f^{\nabla}(b)\right| \int_{0}^{1}|1-2 \widetilde{\rho}(t)| t \tilde{\nabla} t\right] \\
= & \frac{b-a}{4}\left[\left|f^{\Delta}(a)\right| \frac{1}{4}-\left|f^{\Delta}(b)\right| \frac{1}{4}-\left|f^{\nabla}(a)\right| \frac{1}{4}+\left|f^{\nabla}(b)\right| \frac{1}{4}\right] .
\end{aligned}
$$

Corollary 3.3 Suppose $f: \mathbb{Z} \rightarrow \mathbb{R}$ is a function on $[a, b]_{\mathbb{Z}}$ with $a, b \in \mathbb{Z}$ and $a<b$, and $a+b$ an even number. If $\left|f^{\Delta}\right|$ and $\left|f^{\nabla}\right|$ are convex on $[a, b]_{\mathbb{Z}}$, and $\left|f^{\Delta}(a)\right|+\left|f^{\nabla}(b)\right|=\left|f^{\nabla}(a)\right|+\left|f^{\Delta}(b)\right|$, then

$$
\frac{f(a)+f(b)}{2}=\frac{1}{2(b-a)}\left(\int_{a}^{b} f(t) \Delta t+\int_{a}^{b} f(t) \nabla t\right) .
$$

Corollary 3.4 Suppose $f: \mathbb{Z} \rightarrow \mathbb{R}$ is a function on $[a, b]_{\mathbb{Z}}$ with $a, b \in \mathbb{Z}$ and $a<b$, and $a+b$ an even number. If $\left|f^{\Delta}\right|$ and $\left|f^{\nabla}\right|$ are convex on $[a, b]_{\mathbb{Z}}$, then

$$
\frac{f(a)+f(b)}{2}-\frac{1}{2(b-a)}\left(\int_{a}^{b} f(t) \Delta t+\int_{a}^{b} f(t) \nabla t\right) \leq \frac{(b-a)}{16}\left(\left|f^{\Delta}(a)\right|+\left|f^{\nabla}(b)\right|\right) .
$$


Corollary 3.5 Suppose $f: \mathbb{Z} \rightarrow \mathbb{R}$ is a function on $[a, b]_{\mathbb{Z}}$ with $a, b \in \mathbb{Z}$ and $a<b$, and $a+b$ an even number. If $\left|f^{\Delta}\right|$ and $\left|f^{\nabla}\right|$ are convex on $[a, b]_{\mathbb{Z}}$, then

$$
\frac{f(a)+f(b)}{2}-\frac{1}{2(b-a)}\left(\int_{a}^{b} f(t) \Delta t+\int_{a}^{b} f(t) \nabla t\right) \leq \frac{(b-a)}{8}\left(\left|f^{\Delta}(a)\right|+\left|f^{\nabla}(a)\right|+\left|f^{\Delta}(b)\right|+\left|f^{\nabla}(b)\right|\right) .
$$

Theorem 3.6 Suppose $f: \mathbb{Z} \rightarrow \mathbb{R}$ is a convex function on $[a, b]_{\mathbb{Z}}$ with $a, b \in \mathbb{Z}, a<b$ and $a+b$ an even number. Then, the refinement on the right side of the discrete Hermite-Hadamard inequality follows:

$$
\begin{aligned}
& \frac{f(a)+f(b)}{2}-\frac{1}{2(b-a)}\left(\int_{a}^{b} f(t) \Delta t+\int_{a}^{b} f(t) \nabla t\right) \\
& \geq\left|\frac{|f(a)+f(b)|}{2}-\frac{1}{2(b-a)}\left[\int_{[a, b]_{\mathbb{Z}}}|f(t)| \nabla t+\int_{[a, b]_{\mathbf{Z}}}|f(t)| \Delta t\right]\right| \geq 0 .
\end{aligned}
$$

Proof By convexity on $[a, b]_{\mathbb{Z}}$ of $f$, we have

$$
\begin{aligned}
& f(a)+f(b)-f(t a+(1-t) b)-f((1-t) a+t b) \\
& \quad=|f(a)+f(b)-f(t a+(1-t) b)-f((1-t) a+t b)| \\
& \quad \geq|| f(a)+f(b)|-| f(t a+(1-t) b)|-| f((1-t) a+t b)|| \geq 0
\end{aligned}
$$

for all $a, b \in \mathbb{Z}$ and $t \in \mathbb{T}_{[a, b]} \backslash\{0,1\}$. Integrating each side of the inequality on $\mathbb{T}_{[a, b]}$, we obtain

$$
\begin{aligned}
& f(a)+f(b)-\int_{\mathbb{T}_{[a, b]} \subset[0,1]} f(t a+(1-t) b) \tilde{\Delta} t-\int_{\mathbb{T}_{[a, b]} \subset[0,1]} f((1-t) a+t b) \widetilde{\Delta} t \\
& \geq|f(a)+f(b)|-\int_{\mathbb{T}_{[a, b]} \subset[0,1]}|f(t a+(1-t) b)| \widetilde{\Delta} t-\int_{\mathbb{T}_{[a, b]} \subset[0,1]}|f((1-t) a+t b)| \widetilde{\Delta} t \mid .
\end{aligned}
$$

Using the substitution method on time scales (Theorem 2.2), we have

$$
\int_{\mathbb{T}_{[a, b]} \subset[0,1]} f(t a+(1-t) b) \widetilde{\Delta} t=\frac{1}{b-a} \int_{a}^{b} f(t) \nabla t
$$

and

$$
\int_{\mathbb{T}_{[a, b]} \subset[0,1]} f((1-t) a+t b) \widetilde{\Delta} t=\frac{1}{b-a} \int_{a}^{b} f(t) \Delta t,
$$

This completes the proof of the inequality (11).

Corollary 3.7 With the assumptions in Theorem 3.6 and the condition that $f(a+b-x)=f(x)$ for all $x \in[a, b]_{\mathbb{Z}}$, the following inequality is satisfied:

$$
\begin{aligned}
& f(a)-\frac{1}{2(b-a)}\left(\int_{a}^{b} f(t) \Delta t+\int_{a}^{b} f(t) \nabla t\right) \\
& \geq|| f(a)\left|-\frac{1}{2(b-a)}\left[\int_{[a, b]_{\mathbb{Z}}}|f(t)| \nabla t+\int_{[a, b]_{\mathbb{Z}}}|f(t)| \Delta t\right]\right| \geq 0 .
\end{aligned}
$$


Theorem 3.8 With the assumptions in Theorem 3.6, the following inequality is satisfied:

$$
\begin{aligned}
& \frac{1}{2(b-a)}\left(\int_{a}^{b} f(t) \Delta t+\int_{a}^{b} f(t) \nabla t\right)-f\left(\frac{a+b}{2}\right) \\
& \geq\left|\frac{1}{b-a} \int_{[a, b] \mathbb{Z}}\right| \frac{f(x)+f(a+b-x)}{2}|\Delta x-| f\left(\frac{a+b}{2}\right)|| \geq 0 .
\end{aligned}
$$

Proof Fix $t \in \mathbb{T}_{[a, b]} \backslash\{0,1\}$. We define

$$
\begin{aligned}
& x=t a+(1-t) b \\
& y=(1-t) a+t b .
\end{aligned}
$$

It is easy to see that $x, y \in[a, b]_{\mathbb{Z}}$ and $x+y$ is even. Hence, $\frac{1}{2} \in \mathbb{T}_{[x, y]}\left(\right.$ or $\left.\mathbb{T}_{[y, x]}\right)$. Since $f$ is convex on $[x, y]_{\mathbb{Z}}\left(\right.$ or $\left.[y, x]_{\mathbb{Z}}\right)$. We have that

$$
\frac{f(x)+f(y)}{2}-f\left(\frac{x+y}{2}\right) \geq \| \frac{f(x)+f(y)}{2}|-| f\left(\frac{x+y}{2}\right)|| .
$$

Next if we substitute $x$ and $y$ back in $f$, we have

$$
\begin{aligned}
& \frac{f(t a+(1-t) b)+f((1-t) a+t b)}{2}-f\left(\frac{a+b}{2}\right) \\
& \geq|| \frac{f(t a+(1-t) b)+f((1-t) a+t b)}{2}|-| f\left(\frac{a+b}{2}\right) \|
\end{aligned}
$$

for all $t \in \mathbb{T}_{[a, b]} \backslash\{0,1\}$.

Integrating this inequality each side over $\mathbb{T}_{[a, b]}$, we get that

$$
\begin{aligned}
& \int_{\mathbb{T}_{[a, b] \subset[0,1]} f(t a+(1-t) b) \widetilde{\Delta} t+\int_{\mathbb{T}_{[a, b]} \subset[0,1]} f((1-t) a+t b) \widetilde{\Delta t}}^{2}-f\left(\frac{a+b}{2}\right) \\
& \geq\left|\int_{\mathbb{F}_{[a, b]} \subset[0,1]}\right| \frac{f(t a+(1-t) b)+f((1-t) a+t b)}{2}|\widetilde{\Delta} t-| f\left(\frac{a+b}{2}\right)||,
\end{aligned}
$$

Since we have that

$$
\begin{aligned}
& \int_{\mathbb{T}_{[a, b] \subset[0,1]}}\left|\frac{f(t a+(1-t) b)+f((1-t) a+t b)}{2}\right| \widetilde{\Delta} t \\
& =\frac{1}{b-a} \int_{a}^{b}\left|\frac{f(a+b-t)+f(t)}{2}\right| \Delta t,
\end{aligned}
$$

the result follows.

Corollary 3.9 With the assumptions in Theorem 3.6, and the condition that $f(a+b-x)=f(x)$ for all $x \in[a, b]_{\mathbb{Z}}$, the following inequality is satisfied:

$$
\begin{aligned}
& \frac{1}{2(b-a)}\left(\int_{a}^{b} f(t) \Delta t+\int_{a}^{b} f(t) \nabla t\right)-f\left(\frac{a+b}{2}\right) \\
& \geq\left|\frac{1}{b-a} \int_{[a, b]_{\mathbb{Z}}}\right| f(x)|\Delta x-| f\left(\frac{a+b}{2}\right)|| \geq 0 .
\end{aligned}
$$


Open Access This article is distributed under the terms of the Creative Commons Attribution 4.0 International License (http:// creativecommons.org/licenses/by/4.0/), which permits unrestricted use, distribution, and reproduction in any medium, provided you give appropriate credit to the original author(s) and the source, provide a link to the Creative Commons license, and indicate if changes were made.

\section{References}

1. Atıcı, F.M.; Yaldiz, H.: Convex functions on discrete time domains. Can. Math. Bull. 59(2), 225-233 (2016)

2. Bohner, M.; Peterson, A.: Dynamic Equations on Time Scales. An Introduction with Applications. Birkhauser, Boston (2001)

3. Chu, Y.M.; Adil Khan, M.; Khan, T.U.; Ali, T.: Generalizations of Hermite-Hadamard type inequalities for MT-convex functions. J. Nonlinear Sci. Appl. 9(6), 4304-4316 (2016)

4. Chu, Y.M.; Wang, G.D.; Zhang, X.H.: Schur convexity and Hadamard's inequality. Math. Inequal. Appl. 13(4), 725-731 (2010)

5. Dragomir, S.S.; Pearce, C.E.M.: Selected Topics on Hermite-Hadamard Inequalities and Applications. RGMIA Monographs. Victoria University, Footscray (2000)

6. Dragomir, S.S.: Refinements of the Hermite-Hadamard inequality for convex functions. Tamsui Oxf. J. Math. Sci. 17(2), 131-137 (2001)

7. Dragomir, S.S.; Agarwal, R.P.: Two inequalities for differentiable mappings and applications to special means of real numbers and to trapezodial formula. Appl. Math. Lett. 11(5), 91-95 (1998)

8. Eloe, P.W.; Sheng, Q.; Henderson, J.: Notes on crossed symmetry solutions of the two-point boundary value problems on time scales. J. Differ. Equ. Appl. 9(1), 29-48 (2003)

9. Hadamard, J.: Etude sur les propriétés des fonctions entières et en particulier d'une fonction considérée par Riemann. J. Math. Pures Appl. 58, 171-215 (1893)

10. Hermite, Ch: Sur deux limites d'une intégrale définie. Mathesis 3, 82 (1883)

11. Mozyrska, D.; Torres, D.F.M.: The natural logarithm on time scales. J. Dyn. Syst. Geom. Theor. 7(1), 41-48 (2009)

12. Zhang, X.M.; Chu, Y.M.; Zhang, X.H.: The Hermite-Hadamard type inequality of GA-convex functions and its applications. J. Inequal. Appl. 507560, 1-11 (2010)

13. Zhang, X.M.; Chu, Y.M.: Convexity of the integral arithmetic mean of a convex function. Rocky Mt. J. Math. 40(3), 1061$1068(2010)$

Publisher's Note Springer Nature remains neutral with regard to jurisdictional claims in published maps and institutional affiliations. 